\title{
Importance of Health Education for Medical Staff
}

\section{Atef Ali Kloub*}

Central Malaria Lab Section Head, Central Malaria Department, Ministry of Health, UAE

*Corresponding author: Atef Ali Kloub, BSc Public Health, In-Charge of Training and Health Education Department and Central Malaria Lab Section Head, Central Malaria Department, Ministry of Health, UAE, Tel: 971564477982; E-mail: atefali1960@hotmail.com

Received date: November 11, 2015; Accepted date: January 28, 2016; Published date: February 02, 2016

Copyright: () 2016 Kloub AA. This is an open-access article distributed under the terms of the Creative Commons Attribution License, which permits unrestricted use, distribution, and reproduction in any medium, provided the original author and source are credited.

\section{Commentary}

Most of us believe that health education is presented just for general people particularly those who are employed in fields non related to medical aspects. Maybe this is an accurate concept in some situations not in all conditions, so we must know that the main aim for health education is not only to learn others or give them idea about the topic which is the subject of educational health activity, this is a secondary aim because if we considered it as a basic target this will mean that the medical staff may be not in need for education health, therefore each of them, surely know more and more about his job and his task.

I think health education must done for both general people and medical staff individual and for second group the aim is to increase and update their awareness and knowledge in order to get a comprehensive background for many issues they deal with daily and need to change the routine method they used to deal with. While every day there is a new development in methods, materials, information, etc....

For example: The pharmacologist used to describe the patient how to take the doses of treatment (the amount of drug and the time and the period) but in cases like infection of malaria Plasmodium vivax or ovale he should inform the patient why it is necessary to take and complete all the doses of the second antimalarial drug (primaquine) and must explain the personal risk if he ignores taking or completing the treatment which will lead his infection to relapse in future also he will acts as a parasitic carrier and form danger for his community, so if the pharmacologist has no comprehensive background about this topic how he will achieve well, so it is essential for all pharmacologists to know more about malaria disease exactly the mechanism of malaria transmission and the reasons of relapsing cases.

During our daily experience among following up malaria cases, we face fatal errors by physicians may lead to death of some patients when they give them error treatment and when some physicians still have no idea that malaria Plasmodium falciparum is resistant for chloroquine so they give this drug for falciparum cases.

Also physicians must know comprehensive background about the mechanism of malaria transmission and about all lab methods are used for the diagnosis of malaria plasmodium parasite in the lab because some of them for example must not requested for monitoring the patient response for treatment also some kinds of rapid tests results will confuse him when he ask for checking test after the last dose of treatment, so this is wanted from the physician to know what is the pathogenic and the nonpathogenic stages of the parasite in human blood also must know the principle of the diagnostic test how it act to be able to determine for the lab technician which kind or method of test he must use for his request (day today always there is a new diagnostic kits and methods for malarial lab in the market).
Also for the medical lab technicians it is not enough to know how to diagnose the malarial case, but they must know and estimate the risk and what it means and the presence of some stages of the malaria plasmodium parasites in the checked blood film that some of these are dangerous to the patient himself while other stages form a risk for his community and this will not being without understanding exactly the mechanism of malaria transmission to be able to inform the physician what he saw even without a question by the physician (the gametocyte stages in patient blood are the infective form of the parasite for mosquito).

Also the infectious diseases officer must know a comprehensive background about the life cycles for the malaria parasites in both hosts the man and mosquito for the four of malaria plasmodium species which causes infection for human and know the incubation period for each of them which will help him to determine the source of infection and to distinguish between relapse and non-relapse cases when he achieve the epidemiological investigation report for the malarial case.

The epidemiological investigator also must know a lot of data, i.e. entomological information and antimalarial drugs and the malaria endemic countries, this will help him when analyses data. For example entomologically he must know the kinds of all Anopheles species present in his area and around his country and which species of them can transmit malaria.

Also knowledge about antimalarial drugs for each of plasmodium species according to the policy drugs recommended let him able to notify. If there is error in antimalarial drug was given for the patient so he could record a remarkable note when the patient completes the correct dose was given for him. Even though if he didn't respond for the treatment, the patient is still positive for malaria parasite.

The entomological lab technician must know about the stages of parasites which it could found in blood smear to estimate the range of risk with the presence of gametocytes stages in blood smear if the patient residing in an area where there is presence for adult Anopheles mosquitoes from that species which have the ability to transmit malaria disease also he must be always on direct contact with the parasitology lab.

Nurses staff must know some about antimalarial drugs and the impact of its effect so they can notify if patient vomit the drug and record the time of vomiting to determine if it is essential to repeat the dose of treatment. It is important for the nurse to know everything about the main symptoms of the disease to monitor the response of patient for treatment to notify the same to the physician.

In education health presentation about malaria for any medical staff it is essential to them tell them that dealing together with the malarial case must be as a teamwork which will reflect positively and sometimes lead to save a patient life and may the opposite could occur, so as a team for each his own function but also each is a complement to the 
Page 2 of 2

other that may I see or monitor something not under my responsibility but it could be useful for other members, so the members of the team must be cooperative together by sharing data.

The members of team must distinguish between coordination and cooperation from a side and interference factors or negativity competition on the other side so each one must put in his mind that he is in need for the efforts of others which will reflect in positive results for both the patient and community. 